\title{
nature
}

biotechnology

\section{CRISPR catch-up}

\author{
The hegemony of the CRISPR system as a gene-editing therapy is not as assured as its use as a tool in basic \\ research.
}

A s several papers in this issue exemplify, academic researchers continue to find new tricks for CRISPR-associated nucleases. The latest is to fuse Cas 9 to a cytidine deaminase (e.g., see pp. 435, 438, 441 and 475), thereby creating a system that can perform RNA-guided editing at a single base. The frequent disclosure of new and improved Cas9 variants has propelled CRISPR systems to the forefront of basic research, but their supremacy as genome-editing therapeutics remains less clear cut.

CRISPR endonucleases offer the ability to target most genomic targets via facile design of a guide RNA (gRNA), the capacity to multiplex many gene targets simultaneously and the capability to rapidly retarget therapy to a new genomic target (as a malignancy or virus evolves resistance). In contrast, other genome editing systems, like zinc finger nucleases (ZFNs) or transcriptional activator-like effector nucleases (TALENs), require complex and cumbersome protein engineering. Nevertheless, ZFNs or TALENs may be just as effective (or even more effective) for a particular therapeutic target than CRISPR systems. And the former gene editing tools also have a head start in the clinic.

Since CRISPR-Cas9 was first demonstrated to mediate precise genome editing in eukaryotic cells in 2012, the system has taken the academic community by storm. In subsequent years, the number of papers describing CRISPR gene editing has risen from an average of $<1$ per week to 20 per week last year. This is nowhere near the 75 papers per week output for RNA interference (RNAi) in 2016, but it is a great deal more than $\sim 1$ and $\sim 2$ genome-editing papers per week output for ZFNs and TALENs, respectively.

Rapid uptake of CRISPR in academic laboratories has been driven by the ease with which the reagents work in different hands, the wide variety of amenable model systems, and the relative low cost and ease with which gRNAs can be designed to retarget the endonucleases. Yet these attributes don't really matter for current commercial CRISPR therapeutic programs: cost of goods is not a limiting factor for companies developing therapeutics; and as yet there is little need for multiplexing or rapid retargeting of genome editing endonucleases.

Initially, the larger targeting range of ZFNs and especially TALENs was a substantial advantage over CRISPR nucleases, which are constrained by Cas9's requirement for a PAM (protospacer adjacent motif) sequence. This though has become less of an issue as additional CRISPR endonuclease variants with different specificities have been identified from other microbes and as new PAM sequence requirements have been engineered into Cas9. Although comparative data are hard to come by, it is clear that ZFNs, TALENs and CRISPR endonucleases can all knock out genes with efficiencies of $>50 \%$ and that suitable targeting sites can be found (usually in the first exon) of most human genes.

One important difference among the platforms is the nature of the ends that are created when both DNA strands are cut. ZFNs and

TALENs produce 5 ' overhangs that could potentially be exploited for improving incorporation, whereas Cas9 mainly produces blunt ends. Again, the CRISPR field has caught up with the discovery of Cpf1, which does provide $5^{\prime}$ overhangs. Definitive proof that the nature of the cuts provides advantages for precision genome editing for clinical applications remains elusive and many researchers in the field favor inducing single-stranded breaks owing to the reduced risk of creating indels. Compared with ZFNs and TALENs, the specific nature of CRISPR-Cas9 cutting - which involves local unwinding of the duplex and release of single-stranded DNA from the protein-may also provide opportunities for improving homology-direct repair efficiencies unique to Cas9.

High specificity is also at the top of the list of attributes of a clinical genome editing tool, and protein engineering of all three types of endonuclease has been undertaken to address this issue. Worries about potentially harmful off-target effects stem from experience in early gene therapy trials where rare integration events of retroviral vectors led to the development of leukemias. Ultimately, our incomplete understanding of the human genome and the limited sensitivity (at best $0.1 \%$ of all events) of the assays available means that it is prudent to focus on functional assays for tumor formation (or other adverse effects) in a given cell type, a notion that seems to be shared by the regulatory agencies.

Of all the challenges facing therapeutic gene editing, however, delivery remains the hardest to crack. This is one reason why many developers are focusing initially on ex vivo applications (e.g., modifying T cells or hematopoietic stem cells via electroporation). Both DNA and mRNA have been used, but ribonucleoproteins (RNPs) also appear to be an effective modality for Cas9 (e.g., see p. 431). The problem for in vivo applications is the packaging limit $(\sim 4.7 \mathrm{~kb})$ of adeno-associated viral vectors. Packaging regulatory elements, the nucleases themselves and the donor template into a single virus is difficult (though possible) for ZFNs ( $2 \mathrm{~kb}$ per pair), but nigh impossible for Cas $9(3.2-4.2 \mathrm{~kb})$ and TALENs ( $\sim \mathrm{kb}$ per pair). Clearly, the development of minimized endonucleases or combination with lipid nanoparticles or $\mathrm{N}$-acetyl galactosamine conjugates will be a key goal for ongoing efforts.

Compared with developers of ZFNs and TALENs, companies working on CRISPR therapeutics have one massive advantage - a large base of academic researchers with which to collaborate. Of course, industrial R\&D programs will still carry the lion's share of the grueling work translating today's CRISPR research tools into tomorrow's therapies. But having a broad pool of researchers beyond industry to think about new ways of addressing these problems will be a great fillip for the field. As anyone in industry knows, it's always good to have academic colleagues to catch up with. 